\title{
Using a Bent Tube as an Energy Filter for a Positron Beam. Simulations on Determining the Optimum Angle of the Bend
}

\author{
D. DinesCu ${ }^{a, b, *}$ AND N. Djourelov ${ }^{a}$ \\ ${ }^{a}$ University Politehnica of Bucharest, Faculty of Applied Sciences, \\ Splaiul Independentei, no. 313, 060042, Bucuresti, Romania \\ ${ }^{b}$ Extreme Light Infrastructure - Nuclear Physics, Horia Hulubei National Institute for Physics \\ and Nuclear Engineering, 30 Reactorului Str., 077125 Magurele, Ilfov county, Romania
}

One way to perform lifetime measurements at a DC positron beam is to pass positrons through a pulsing device. The compressed $\mathrm{e}^{+}$bunches are accelerated to a desired energy for depth profiling of the studied sample. A fraction of the $\mathrm{e}^{+}$that are backscattered from the sample surface can travel back through the uniform magnetic field that is generated along the central axis of the beam transport lines and reach the accelerator. The backscattered $\mathrm{e}^{+}$that reach the accelerator can be re-accelerated towards the sample and re-implant in it with a delay from the initial implanted $\mathrm{e}^{+}$bunch. These $\mathrm{e}^{+}$that are re-implanted into the sample with a delay cause distortions in the lifetime spectra. A setup which uses a bent tube to act as an energy filter to reduce the effect of the backscattered $\mathrm{e}^{+}$is studied by simulations. The simulations are performed in order to find the optimum geometry for minimum distortions.

DOI: 10.12693/APhysPolA.132.1624

PACS/topics: 41.75.Fr, 78.70.Bj

\section{Introduction}

At the extreme light infrastructure-nuclear physics (ELI-NP) facility, an e beam will be obtained through the mechanism of pair production, by the interaction of a brilliant $\gamma$ beam with a suitable target (convertormoderator assembly). The $\gamma$ beam will create, through the interaction with the convertor-moderator assembly, an intense positron beam [1].

One of the setups that will be built in the first phase of the project is a spectrometer for positron annihilation lifetime spectroscopy (PALS) with slow $\mathrm{e}^{+}$. As for any experiment that performs lifetime measurements, a start and a stop signal is needed in order to obtain a lifetime spectrum. For the ELI-NP $\mathrm{e}^{+}$beamlines, a pulsing device, based on the principle of the chopping and bunching techniques, has been designed and simulated [2]. The start signal necessary for the PALS measurements will be derived from the pulsing electronics.

In order to perform depth profiling measurements, the $\mathrm{e}^{+}$will be accelerated to a desired energy of up to $30 \mathrm{keV}$ at the exit of the main buncher. When the accelerated $\mathrm{e}^{+}$hit the studied sample, a fraction of them will be backscattered. These backscattered $\mathrm{e}^{+}$, guided by the uniform magnetic field generated along the central axis of the transport lines, can travel back to the accelerator and be re-accelerated towards the sample, thus implanting in it with a delay. The gammas arising from the annihilation of the delayed $\mathrm{e}^{+}$will cause distortions in the PALS spectrum as satellite peaks. The described

*corresponding author; e-mail: doru.dinescu@eli-np.ro effect is well known, and several solutions have been applied worldwide: using a $\boldsymbol{E} \times \boldsymbol{B}$ filter [3], designing an accelerator-decelerator structure [4], and using $45^{\circ}$ bent tube equipped with steering coils to act as an energy filter [5]. For the designed PALS spectrometer which will be implemented at ELI-NP, using a bent tube as an energy filter will be utilised for minimizing the distortions in the PALS spectra.

The aim of the study reported in the present paper is to determine the optimum parameters of the bend tube energy filter for minimum PALS spectra distortions.

\section{Simulations}

In order to simulate the designed setup, Comsol Multiphysics ${ }^{\circledR}$ and Geant4 softwares are used. Comsol is used for generating the magnetic and electric fields, but due to the fact that it does not have the Compton scattering process implemented into it, Geant 4 is used for a realistic simulation of the positron backscattering.

The magnetic field necessary for guiding the positron beam along the central axis of the beam transport lines is simulated in Comsol by the use of a series of multi-turn Helmholtz coils. It was cross-checked in Comsol that the close to uniform ( $60 \pm 2 \mathrm{G}$ on the central axis of the beam transport lines) magnetic field which is obtained, keeps the $\mathrm{e}^{+}$beam confined to the axis, and that the positrons hit the target central region. Comsol is also used for generating the electric field needed for $\mathrm{e}^{+}$acceleration to energies of $2-30 \mathrm{keV}$. The accelerator is positioned at the exit of the main buncher, and at a distance of $720 \mathrm{~mm}$ (along the axis) away from the target. The geometry of the Faraday cage, with an internal diameter of $116 \mathrm{~mm}$, shown in Fig. 1 is composed of a bent part with a length 
which was kept fix at $450 \mathrm{~mm}$ at all the studied angles followed by a straight part with a length of $270 \mathrm{~mm}$. The acceleration field is obtained by applying a potential $U_{a c c}$ equally spread on the nine graded electrodes, with holes of $60 \mathrm{~mm}$ in diameter. After the $\mathrm{e}^{+}$are accelerated to the desired energy, they travel the distance to the target through the Faraday cage which is kept at the same potential $U_{a c c}$, in order for it to act as a drift region.

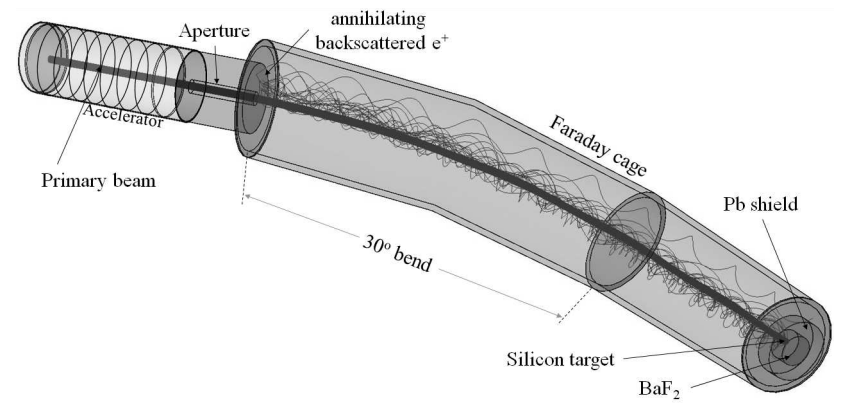

Fig. 1. Geometry of the accelerator and the Faraday cage composed of bent and straight part. The example is for a $30^{\circ}$ bend with an aperture of $D=15 \mathrm{~mm}$. The displayed trajectories are obtained by Geant 4 for a $2 \mathrm{keV}$ $\mathrm{e}^{+}$beam.

The values for the magnetic and electric fields obtained from Comsol are taken in a 3D grid like pattern and exported as an ASCII file. The file is properly prepared so that it can be imported into Geant4, a software which presents al the physics models necessary for conducting a $\mathrm{e}^{+}$backscattering study.

An identical geometry as the one from Comsol is also constructed in Geant4. This is done in order to make sure that the electric and magnetic fields that are imported, perfectly overlap. For each case that was studied this fact has been crosschecked by monitoring the position where the $\mathrm{e}^{+}$beam hits the target. Various low energy physics models which include ionization, bremsstrahlung and multiple scattering, that are implemented in Geant4 are tested for the simulations. The results obtained are crosschecked, and all of the physics models give comparable data. G4EmLivermorePhysics model has been chosen to complete the study due to the time per studied case factor (simulations were performed approximately $25 \%$ faster than for the other low energy physics models).

The simulations are performed with the use of silicon as a target. The $\mathrm{e}^{+}$backscattering coefficient increases with the atomic number of the target material [6]. Silicon, having $Z=14$, has a relatively low $\mathrm{e}^{+}$backscattering coefficient but we chose it as a target in order to underline the importance of the studied effect. For each simulation an instantaneous shot of $10^{6} \mathrm{e}^{+}$is released at time zero towards the sample, and accelerated to the desired energy by the electric field of the accelerator.

In Fig. 2a, the time of annihilation within the target for $\mathrm{e}^{+}$accelerated to $E_{+}=5 \mathrm{keV}$ is presented. The very sharp peak at $\approx 25$ ns corresponds to those $\mathrm{e}^{+}$which are directly implanted into the target. It can be seen from

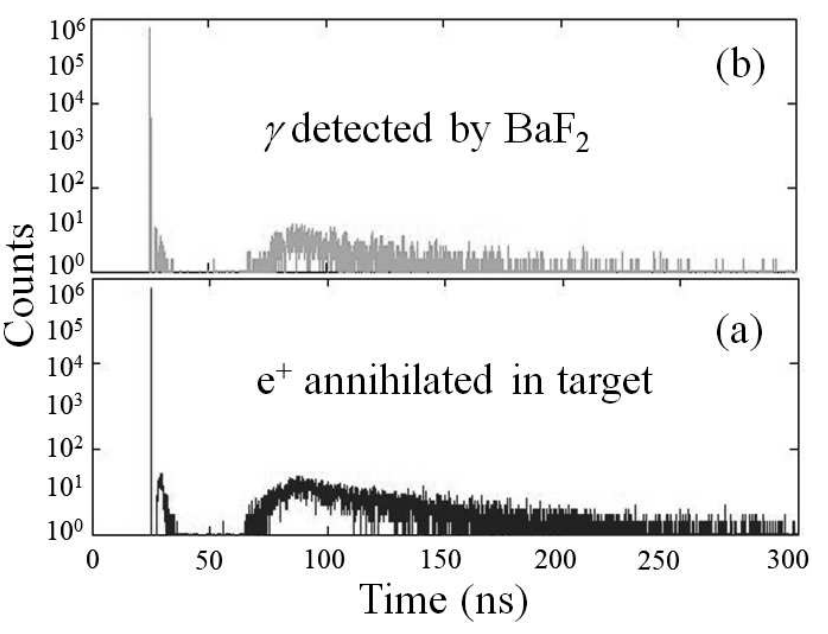

Fig. 2. Histograms of the time of (a) the $\mathrm{e}^{+}$annihilation within the target and (b) the gamma rays interaction with the $\mathrm{BaF}_{2}$ detector obtained by Geant 4 for a $\mathrm{e}^{+}$beam accelerated to $E_{+}=5 \mathrm{keV}$ and $0^{\circ}$ bent tube (i.e. without energy filter).

the very broad peak which starts at $\approx 75$ ns that, even with the use of a silicon target, $\mathrm{e}^{+}$will be backscattered and reflected back. The broadness of the peak is due to the wide distribution of the normal component of the energy of the backscattered $\mathrm{e}^{+}$. The small peak between the sharp and the broad peaks is caused by those $\mathrm{e}^{+}$ which are backscattered from the sample at large angles. These $\mathrm{e}^{+}$hit the walls of the Faraday cage close to the sample and are reflected back onto its surface. This effect only appears for $\mathrm{e}^{+}$energies higher than $2 \mathrm{keV}$, has been previously observed and it only affects the shortlived components, as opposed to the effect caused by the re-accelerated $\mathrm{e}^{+}$which affect the long-lived components. Several solutions have been devised in order to eliminate the distortions caused to the short-lived components. These include the use of a sample chamber with a large diameter or to increase the strength of the guiding magnetic field to reduce the Larmor radii [7].

Behind the target, a $\mathrm{BaF}_{2}$ detector is implemented in Geant4 (see Fig. 1). This is done in order to detect the $511 \mathrm{keV}$ gamma rays which arise from the $\mathrm{e}^{+}$ annihilation. In order for the obtained data to be as close as possible to the case of a real experiment, the detector discounts all the gamma quanta which deposit less than $300 \mathrm{keV}$. Figure $2 \mathrm{~b}$ presents the histogram of the time of the gamma rays interaction with the $\mathrm{BaF}_{2}$ detector obtained by Geant4. Geant4 does not take into account the $\mathrm{e}^{+}$lifetime in a material. In order to simulate PALS spectra, we convolute a resolution function with the probabilities of positrons annihilating from a discrete number of states by the formula: $y(t)=R(t) \otimes\left(N_{t} \sum_{i=1}^{n}\left(I_{i} / \tau_{i}\right) \exp \left(-t / \tau_{i}\right)+B\right)$, where $\tau_{i}$ is the lifetime of the $i$-th state(component) with intensity $I_{i}, N_{t}$ is the total counts and $B$ is the background. The data shown in Fig. $2 \mathrm{~b}$ represents an example of the res- 
olution function. For each studied case the corresponding resolution function is simulated. Our choice for the components of the simulated PALS spectra was lifetimes $\left[\tau_{1}, \tau_{2}, \tau_{3}, \tau_{4}\right]=[0.1,0.5,3,20] \mathrm{s}$ with corresponding intensities $\left[I_{1}, I_{2}, I_{3}, I_{4}\right]=[10,60,10,20] \%$. The typical resolution of a $\mathrm{BaF}_{2}$ detector is also taken into account. Figure 3 presents the simulated PALS spectra for $\mathrm{e}^{+}$accelerated to $E_{+}=5 \mathrm{keV}$ and $0^{\circ}$ bent tube for two aperture diameters: (a) $D=60 \mathrm{~mm}$ and (b) $D=15 \mathrm{~mm}$. In the case of the wide aperture, the distortion caused by the backscattered $\mathrm{e}^{+}$can be clearly seen in the interval 50-150 ns (Fig. 3a). In the case of the narrow aperture (Fig. 3b) the distortion effect is barely visible. The importance of using a narrow aperture is already discussed in [8] and the data we discuss below will concern only the narrow aperture case.

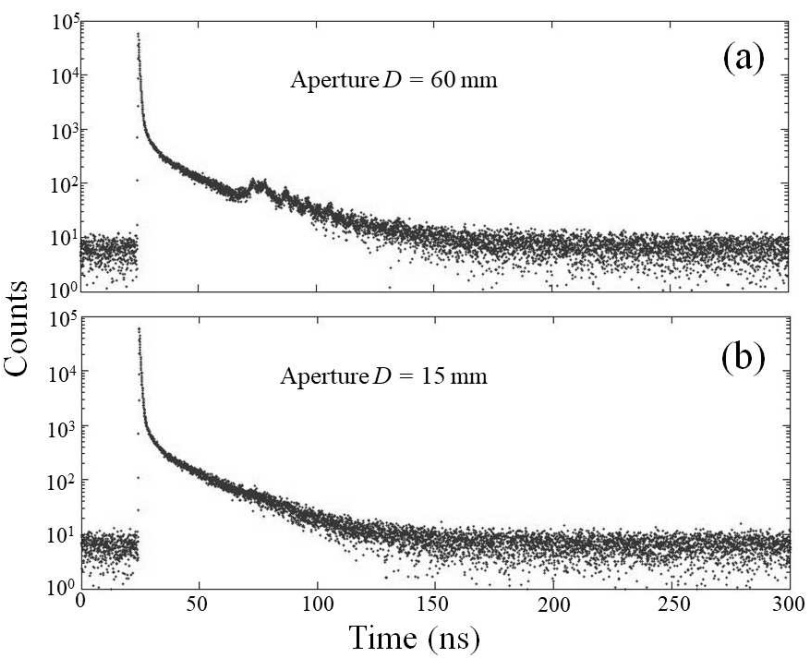

Fig. 3. Simulated PALS spectra for an $\mathrm{e}^{+}$beam accelerated to $E_{+}=5 \mathrm{keV}$ and $0^{\circ}$ bent tube for aperture diameters: (a) $D=60 \mathrm{~mm}$ and (b) $D=15 \mathrm{~mm}$.

In order to quantify the barely visible distortions, the simulated spectra are analysed by the LT9 software [9] and the best fit parameters are compared with the model parameters. The averaged deviations from the model are calculated by the formula: $\Delta=100 \times \sum_{i}\left|\left(P_{i}-P_{i}^{f i t}\right) / P_{i}\right|$, where $P=\left[\tau_{1}, \tau_{2}, \tau_{3}, \tau_{4}, I_{1}, I_{2}, I_{3}, I_{4}\right]$.

\section{Results and discussion}

The trajectories of $2 \mathrm{keV}$ incident $\mathrm{e}^{+}$and the event of $\mathrm{e}^{+}$backscattering with 1000 incident particles for a $30^{\circ}$ bend geometry and an aperture of $D=15 \mathrm{~mm}$ as obtained by Geant4 is shown in Fig. 1. It is seen that the backscattered $\mathrm{e}^{+}$are deflected from the beam axis.

The idea of adding a bent tube right before the sample is for it to act as an energy filter. The trajectories of the incident $\mathrm{e}^{+}$follow the axis of the beam line by a proper adjustment of the magnetic field generated by a pair of correction coils. The $\mathrm{e}^{+}$are backscattered from the sample with a lower energy than the incident energy

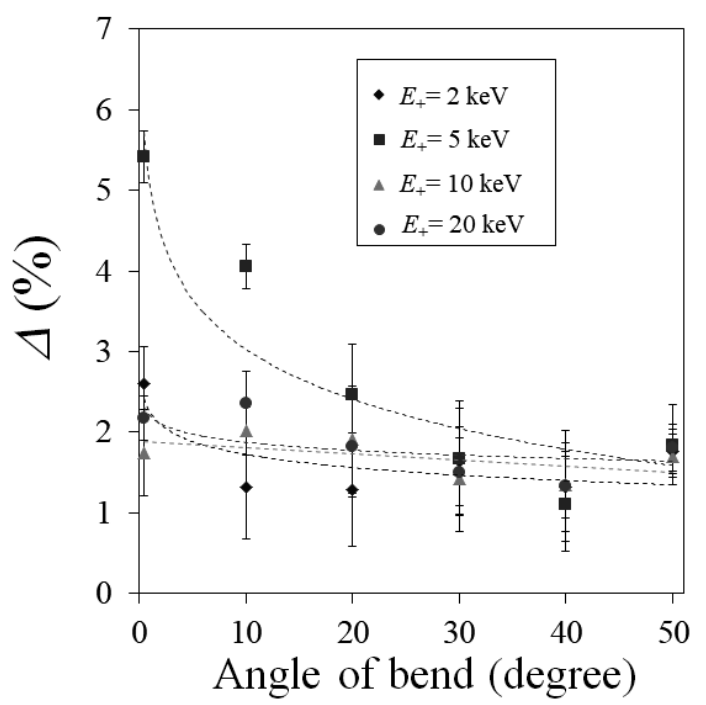

Fig. 4. The averaged deviations of the best fit parameters of a simulated spectrum from the model spectrum for a set of bent tube angles and $\mathrm{e}^{+}$energies. The trend lines are added to guide the eye.

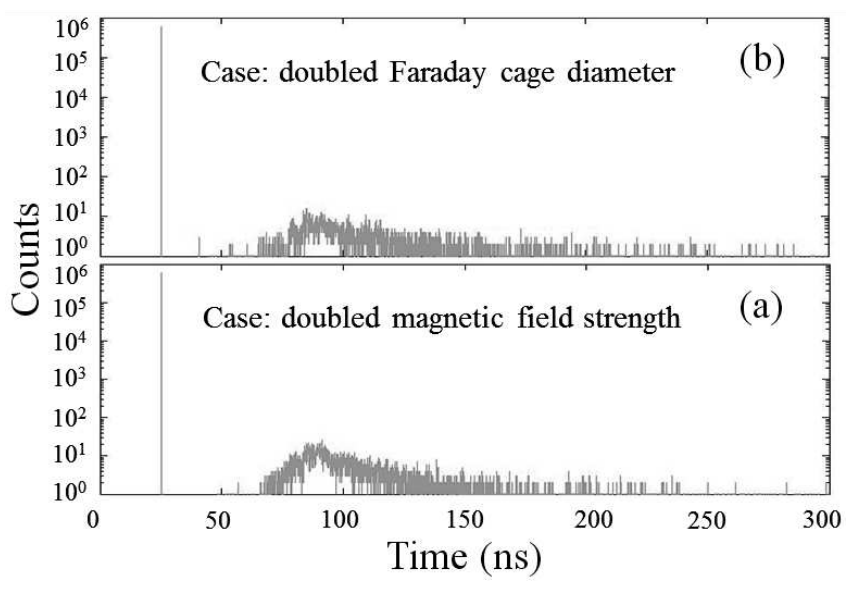

Fig. 5. Gamma rays detected by the $\mathrm{BaF}_{2}$ detector in two cases: (a) of doubled magnetic field strength and (b) of doubled diameter of the Faraday cage obtained by Geant 4 for a $\mathrm{e}^{+}$beam accelerated to $E_{+}=5 \mathrm{keV}$ and $0^{\circ}$ bent tube (i.e. without energy filter).

which prevents them from following the axis in their return path. Deflected, they do not reach the accelerator and eventually their annihilation occurs far from the $\mathrm{BaF}_{2}$ detector.

We performed a series of simulations in order to find the optimum geometry for minimum distortions. The cases which are analysed include four incident energies $E_{+}=[2,5,10,20] \mathrm{keV}$. For each of the four energies, the studies for bent angles in the interval $0^{\circ}-50^{\circ}$ are performed. The averaged deviations of the best fit parameters of a simulated spectrum from the model spectrum for a set of bent tube angles and $\mathrm{e}^{+}$energies are shown in Fig. 4. The averaged deviations gradually decrease with 
the increase of the bend angle. A saturation at $\Delta<2 \%$ is observed for bent angles $\geq 30^{\circ}$. This affirms that the designed energy filter will work for a reduction of the distortions induced by backscattered $\mathrm{e}^{+}$.

At high $E_{+}$the $\mathrm{e}^{+}$which are backscattered from the sample at large angles hit the Faraday cage side wall with a higher probability due to the larger Larmor radii of their trajectories. As it was discussed in Sect. 2, the solution to reduce the above effect is either to increase the diameter of the Faraday cage or to decrease the Larmor radius by increasing the strength of the guiding magnetic field. Figure 5 shows the $\gamma$ rays detected by the $\mathrm{BaF}_{2}$ detector in two cases: of doubled diameter of the Faraday cage and of doubled magnetic field strength for the same $E_{+}$and geometry used for the generation of the data in Fig. 2a. A comparison between the data from Fig. 5 and Fig. 2a shows that the small peak, right after the sharp peak seen in Fig. 2a, is eliminated which can further reduce the PALS spectra distortions.

\section{Conclusions}

The performance of the setup which uses a bent tube to act as an energy filter was studied by the help of Comsol and Geant4 simulations. The time histograms of the gammas detected by a $\mathrm{BaF}_{2}$ detector obtained by Geant4 were used as resolution functions for generation of PALS spectra. The best fits of the spectra were compared to the model spectrum. The distortions in the generated spectra were quantified by the averaged deviations of the best fit parameters from the model. The analysis of the averaged deviations demonstrated that the bent tube efficiently reduces the effect of the backscattered $\mathrm{e}^{+}$. For minimum distortions the $3^{\circ}$ bend can be considered as an optimal solution.

\section{Acknowledgments}

The support from the ELI-NP Phase II, a project cofinanced by the Romanian Government and the European Union through the European Regional Development Fund - the Competitiveness Operational Program (1/07.07.2016, COP, ID 1334) is acknowledged.

\section{References}

[1] N. Djourelov, C. Hugenschmidt, S. Balascuta, V. Leca, A. Oprisa, C. Piochacz, C. Teodorescu, C.A. Ur, Rom. Rep. Phys. 68, S735 (2016).

[2] N. Djourelov, D. Dinescu, J. Phys. Conf. Ser. 791, 012010 (2017).

[3] W. Egger, P. Sperr, G. Kögel, G. Dollinger, Phys. Status Solidi C 4, 3969 (2007).

[4] A. Pelli, A. Laakso, K. Rytsölä, K. Saarinen, Appl. Surf. Sci. 252, 3143 (2006).

[5] M. Jungmann, J. Haeberle, R. Krause-Rehberg, W. Anwand, M. Butterling, A. Wagner, J.M. Johnson, T.E. Cowan, J. Phys. Conf. Ser. 443, 012088 (2013).

[6] J. Makinen, S. Palko, J. Martikainen, P. Hautojarvi, J. Phys. Condens. Matter 4, L503 (1992).

[7] K. Fallström, T. Laine, Appl. Surf. Sci. 149, 44 (1999).

[8] D. Dinescu, N. Djourelov, U.P.B. Sci. Bull., submitted for publication.

[9] J. Kansy, Nucl. Instrum. Methods Phys. Res. A 374, 235 (1996). 